\title{
Energy Consumption Using Network Stability And Multi-hop Protocol For Link Efficiency in Wireless Body Area Networks
}

\author{
Mohd Kaleem ${ }^{1}$, Dr. R. P. Mahapatra ${ }^{2}$ \\ Department, Computer Science and Engineering SRM University India ${ }^{l}$ \\ Head of the Department, Computer Science and Engineering SRM University India ${ }^{2}$
}

\begin{abstract}
In this paper, we propose a minimum, power efficient and max throughput routing protocol for Wireless Body Area Networks (WBANs). We use multi-hop topology to achieve minimum energy consumption and longer network lifetime. We propose a cost function to select forwarder node. Proposed cost function selects a forwarder node which has high residual energy and minimum distance to sink. Residual energy parameter balances the energy consumption among the sensor nodes while distance parameter ensures successful packet delivery to sink. Simulation results show that our proposed protocols maximize the network stability period and nodes stay alive for longer period. Longer stability period contributes high packet delivery to sink which is major interest for continuous patient monitoring.
\end{abstract}

Keywords: Wireless body Area Network, Cost Function, and Residual Energy.

\section{Introduction}

Wireless Sensor Networks (WSNs) are used to monitor certain parameters in many applications like environment monitoring, habitant monitoring, [1] battle field, agriculture field monitoring and smart homes. These wireless sensors aredispersed in sensing area to monitor field. WBAN is new emerging sub-field of WSN. A key application of WBAN is health monitoring. Wireless sensors are placed on the humanbody or implanted in the body to monitor vital signs like blood pressure, body temperature, heart rate, glucose level etc. Use of WBAN technology to monitor health parameters significantlyreduces the expenditures of patient in hospital. With the help of WBAN technology, patients are monitored at home for longer period. Sensors continuously sense data and forwardto medical server. In WBANs, sensor nodes are operated with limited energy source. It is required to use minimum power for transmittingdata from sensor nodes to sink. One of the major obstacles in WBAN is to recharge the batteries. An efficient routing protocolis required to overcome this issue of recharging batteries.Many energy efficient routing protocols are proposed in WSNtechnology. However, WSNs and WBANs have different architectures, applications and operate in different conditions. It is impossible to port WSN routing protocols to WBAN. Therefore, energy efficient routing protocol forWBAN is required to monitor patients for longer period.

We propose a high throughput, reliable and stable routing protocol for WBAN. We deploy sensor nodes on the body at fixed places. We place sink at waist. Sensors for ECG and Glucose level are placed near the sink. Both these sensors have critical data of patient and required minimum attenuation, high reliability and long life therefore; these sensors always transmit their data directly to sink. Other sensors follow their parent node and transmit their data to sink through forwardernode. It saves energy of nodes and network works for longer period.

\section{Wban Infrastructure}

A WBAN consists of in-body and on-body nodes that continuously monitor patient's vital information for diagnosis and prescription. Some on-body nodes can also be used for multimedia andgaming applications. A WBAN uses Wireless Medical Telemetry Services (WMTS), unlicensedIndustrial, Scientific, and Medical (ISM), Ultrawideband (UWB), and Medical Implant Communications Service (MICS) bands for data transmission. WMTS is a licensed band used for medical telemetry system. Federal CommunicationCommission (FCC) urges the use of WMTS for medical applications due to fewer interfering sources. However, only authorized users such as physicians and trained technicians are eligible to use this band. Furthermore, the restricted WMTS(14 MHz) bandwidth cannot support video and voice transmissions. The alternative spectrum for medical applications is to use $2.4 \mathrm{GHz}$ ISM band that includes guard bands to protect adjacentchannel interference. A licensed MICS band (402-405 MHz) is dedicated to the implant communication. Fig.1.1 shows a WBAN infrastructure for medical and nonmedical applications. As can be seen in the figure, the WBAN traffic is categorized into On-demand, Emergency, and Normal traffic. On-demand trafficis initiated by the coordinator or doctor to acquirecertain information, mostly for the purpose of diagnostic recommendations. This is further divided into continuous (in case of surgical events)and discontinuous (when occasional information isrequired). Emergency traffic is initiated by thenodes when they exceed a predefined threshold andshould be 
accommodated in less than one second. This kind of traffic is not generated on regular intervals and is totally unpredictable. Normal traffic is the data traffic in a normal condition with no time critical and on-demand events. This includes unobtrusive and routine health monitoring of a patient and treatment of many diseases such as gastrointestinal tract, neurological disorders, cancer detection, handicap rehabilitation, and the most threatening heart disease. The normal data is collected and processed by the coordinator. The coordinator contains a wakeup circuit, a main radio, and a bridging function, all of them connected to a data interface. The wakeup circuit is used to accommodate on demand and emergency traffic. The bridging function is used to establish a logical connection between different nodes working on different frequency bands. The coordinator is further connected to telemedicine, game, and medical servers for relevant recommendations.

\section{Related Work}

InWBAN technology, large numbers of routing schemes areproposed. In this section, we present some proposed routingprotocols. In [5] the author presented a thermal aware routingprotocol. Each node selects a minimum hop rout to sink. Whena parent node gets heated, the children nodes select anotheroptimal route.Latreet al. proposed A Secure Low-Delay Protocol forMulti-hop Wireless Body Area Networks (CICADA) routingprotocol which consists of a spanning tree structure [6]. CICADAused Time Division Multiple Access (TDMA) protocolto schedule transmission of nodes. The nodes near the root actas forwarder nodes or parent nodes, these nodes collect datafrom their associated children nodes and relay to sink. Dueto extra traffic load of children nodes on parent nodes causesparent nodes to deplete their energy fast.In [7] Quwaideret al. presented a routing protocol whichtolerates to changes in network. They used store and forward mechanism to increase the likelihood of a data packet to reachSuccessfully to sink node. Each sensor node has the capabilityto store a data packet. In source to destination route, each nodestores data packet and transmits to next node. Storing a datapacket and then retransmitting causes more energy to consumeand longer end to end delay.Ehyaiet al. [8] proposed a solution to minimize energy consumption. They deploy some non-sensing, dedicated nodes with additional energy source. This technique minimizes energy consumption of nodes and enhances the network lifetime, however, additional hardware required for relay nodes increase the cost of the network.A clustering based protocol A Self-Organization Protocol for Body Area Networks (ANYBODY) is proposed in [9]. The objective of this protocol is to restrict the sensor nodes to transmit data direct to sink. It improves the efficiency of network by changing the selection criteria of CHs. In [10] Nabiet al. proposed a protocol similar to store and forward mechanism. They integrate this store and forward scheme with Transmit Power Adaption (TPA). To control transmission power consumption, all nodes know their neighbours. Nodes transmit data with minimum power and with a stable link quality. A similar method was proposed by Guoet al. in [11]. They also used Transmission Power Control (TPC) scheme as Nabiet al. proposed. When link quality of a node decreased, an Automatic Repeat Request (ARR) is transmitted and retransmit the drop packet. Retransmission of lost packet increasesthe throughput of the network with the expense of energyconsumption.Tsouriet al. in [12], [13] used creeping waves to relaydata packet. They proposed this protocol to minimize energyconsumption of nodes while keeping the reliable on body link. Authors in [14], [15] analyze delay in WBANs and differentmedium access techniques forWBAN. In [16] author proposeda delay tolerant protocol. They compare their protocol withdifferent existing protocols.

\section{Motivation}

Wireless Body Area Sensors are used to monitor humanhealth with limited energy resources. Different energyefficient routing schemes are used to forward data from bodysensors to medical server. It is important that sensed dataof patient reliably received to medical specialist for furtheranalysis. In [17] author presented a opportunistic protocol.Proposed scheme facilitate mobility at cost of low throughputand additional hardware cost of relay node. They deploy sinkat wrist. Whenever sink node goes away from transmissionrange of nodes, it uses a relay node which collect data fromsensor nodes. In opportunistic protocol, whenever patientmoves his hands, the wireless link of sink with sensor nodesdisconnects. Link failure consumes more power of sensornodes and relay node also more packets will drop, whichcauses important and critical data to loss.To minimize energy consumption and to increase thethroughput, we propose a new scheme. Our contributionincludes:

- Our proposed scheme achieves a longer stability period.Nodes stay alive for longer period and consume minimumenergy.

- Large stability period and minimum energy consumptionof nodes, contribute to high throughput. 


\section{Radio Model}

Many radio models are proposed in literature. We use firstorder radio model proposed in [18]. This radio model considers $\mathrm{d}$, the separation between transmitter and receiver and $\mathrm{d} 2$, theloss of energy due to transmission channel. First order radiomodel equations are given as.

$$
\begin{gathered}
\operatorname{ETx}(\mathrm{k}, \mathrm{d})=\operatorname{ETx}-\operatorname{elec}(\mathrm{k})+\operatorname{ETx}-\operatorname{amp}(\mathrm{k}, \mathrm{d}) \\
\operatorname{ETx}(\mathrm{k}, \mathrm{d})=\operatorname{ETx}-\operatorname{elec} \times \mathrm{k}+\operatorname{Eamp} \times \mathrm{k} \times \mathrm{d} 2(1) \\
\operatorname{ERx}(\mathrm{k})=\operatorname{ERx}-\operatorname{elec}(\mathrm{k}) \operatorname{ERx}(\mathrm{k})=\operatorname{ERx}-\mathrm{elec} \times \mathrm{k} \\
\operatorname{ERx}(\mathrm{k})=\operatorname{ERx}-\operatorname{elec} \times \mathrm{k}(2)
\end{gathered}
$$

Where ETxis the energy consumed in transmission, ERxisthe energy consumed by receiver, $E T x-e l e c a n d E R x-e l e c a r e t h e$ energies required to run the electronic circuit of transmitterand receiver, respectively. Eampis the energy required foramplifier circuit, while $\mathrm{k}$ is the packet size.

In WBAN, the communication medium is human body whichcontributes attenuation to radio signal. Therefore, we add pathloss coefficient parameter $\mathrm{n}$ in radio model. Equation 2 oftransmitter can be rewritten as.

$$
\operatorname{ETx}(\mathrm{k}, \mathrm{d})=\operatorname{Eelec} \times \mathrm{k}+\operatorname{Eamp} \times \mathrm{n} \times \mathrm{k} \times \mathrm{dn}(3)
$$

The energy parameters given in equation 3 depend on thehardware. We consider two transceivers used frequently inWBAN technology. The Nordic nRF 2401A is a singlechip, low power transceiver and other transceiver is ChipconCC2420. The bandwidth of both transceivers is $2.4 \mathrm{GHz}$.We use the energy parameter of The Nordic nRF 2401Atransceiver because it consumes less power than ChipconCC2420. The energy parameters for this transceiver are givenin Table 1.

\section{Nsamp: Protocol Detail}

In this section, we present a routing protocol forWBANs. The limited numbers of nodes in WBANs giveopportunity to relax constraints in routing protocols. Keepingrouting constrains in mind, we improve the network stabilityperiod and throughput of the network. Next subsections givedetail of the system model and detail of NSAMPprotocol.

\section{A. System Model}

In this scheme, we deploy eight sensor nodes on humanbody. All sensor nodes have equal power and computationcapabilities. Sink node is placed at waist. Node 1 is ECGsensor and node 2 is Glucose sensor node. These two nodestransmit data direct to sink. Fig 1 shows the placement ofnodes and sink on the human body.

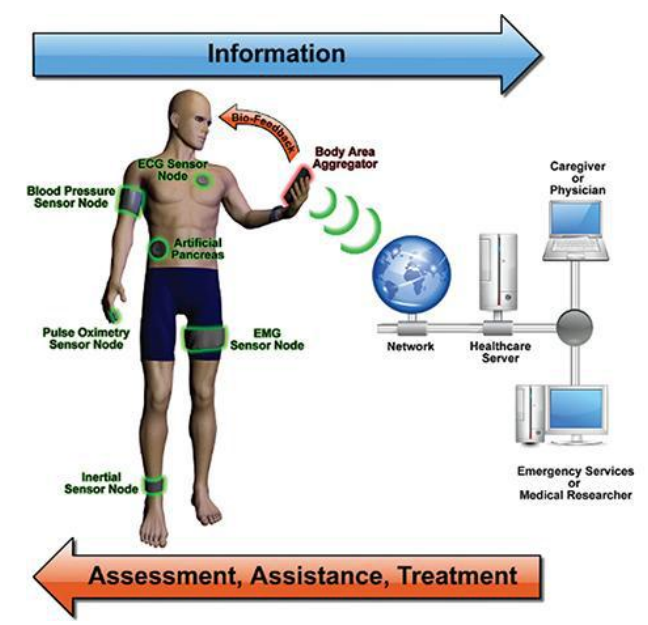

Fig: 1 Model of (WBAN)

\begin{tabular}{|l|l|l|l|}
\hline Parameters & nRF 2401A & CC2420 & Units \\
\hline DC Current(Tx) & 10.5 & 17.4 & $\mathrm{Ma}$ \\
\hline DC Current(Rx) & 18 & 19.7 & $\mathrm{~mA}$ \\
\hline Supply Voltage(min) & 1.9 & 2.1 & $\mathrm{~V}$ \\
\hline${ }^{\mathrm{E}} \mathrm{tx}-\mathrm{elec}$ & 16.7 & 96.9 & $\mathrm{~nJ} / \mathrm{bit}$ \\
\hline${ }^{\mathrm{E}} \mathrm{rx}-\mathrm{elec}$ & 36.1 & 172.8 & $\mathrm{~nJ} / \mathrm{bit}$ \\
\hline Eamp & $1.97 \mathrm{e}-9$ & $2.71 \mathrm{e}-7$ & $\mathrm{j} / \mathrm{b}$ \\
\hline
\end{tabular}



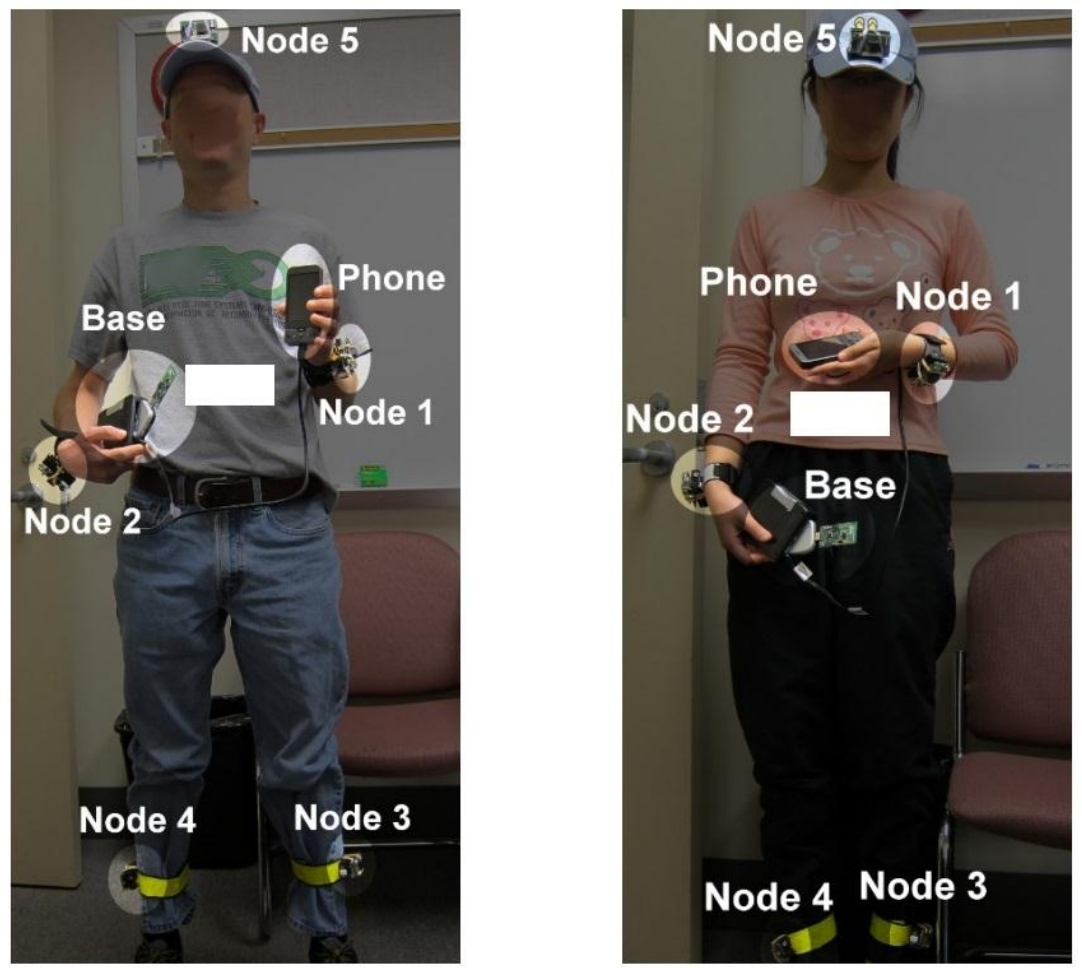

Fig. 2: Node deployment

\section{B. Initial Phase}

In this phase, sink broadcast a short information packet which contains the location of the sink on the body. After receiving this control packet, each sensor node stores the location of sink. Each sensor node broadcasts an information packet which contains node ID, location of node on body andits energy status. In this way, all sensor nodes are updated with the location of neighbours and sink.

\section{Selection of next hop}

In order to save energy and to enhance network throughput, we proposed a multi hop scheme for WBAN. In this section, we present selection criteria for a node to become parent node or forwarder. To balance energy consumption amongsensor nodes and to trim down energy consumption of network, NSAMP protocol elects new forwarder in each round. Sink node knows the ID, distance and residual energy status of the nodes. Sink computes the cost function of all nodes and transmit this cost function to all nodes.On the basis of this cost function, each node decides whether to become forwarder node or not. If $i$ is number of nodes than cost function of $\mathrm{i}$ nodes is computed as follows:

$$
\text { C.F (i) }=\mathrm{d}(\mathrm{i}) / \mathrm{R} . \mathrm{E}(\mathrm{i})
$$

Where di is the distance between the node $i$ and sink, R.Eiis the residual energy of node $i$ and is calculated by subtracting the current energy of node from initial total energy. A node with minimum cost function is preferred as a forwarder. All the neighbor nodes stick together with forwarder node and transmit their data to forwarder. Forwarder node aggregates data and forward to sink. Forwarder node has maximum residual energy and minimum distance to sink; therefore, it consumes minimum energy to forward data to sink. Nodes for ECG and Glucose monitoring communicate direct to sink and do not participate in forwarding data.

\section{Scheduling}

In this phase, forwarder node assigns a Time Division Multiple Access (TDMA) based time slots to its children nodes. All the children nodes transmit their sensed data to forwarder node in its own scheduled time slot. When a node has no data to send, it switches to idle mode. Nodes wake up only at its transmission time.Scheduling of sensor nodes inimize the energy dissipation of individual sensor node.

\section{Performance Metrics}

We evaluated key performance metrics for proposed protocol.Definition of performance metrics is given in followingsubsections.

1) Network lifetime: It represents the total network 
operation time till the last node die.

2) Stability period: Stability period is the time span ofnetwork operation till the first node die. The time period afterthe death of first node is termed as unstable period.

3) Throughput: Throughput is the total number of packetssuccessfully received at sink.

4) Residual Energy: In order to investigate the energyconsumption of nodes per round, we consider residual energyparameter to analyze energy consumption of network.

5) Path Loss: Path loss is the difference between thetransmitted power of transmitting node and received powerat receiving node. It is measured in decibels $(\mathrm{dB})$.

\section{Simulation Results And Analysis}

To evaluate proposed protocol, we have conducted an extensiveset of experiments using MATLAB R2013. We studiedthe performance of theNSAMP protocol by comparisons with the existing protocol ATTEMPT.

\section{A. Network life time}

Fig 2 shows the average network lifetime of proposed scheme. The proposed new cost function to elect forwarder node play an important role to balance the energy consumption among the sensor nodes. New forwarder in each round is selected based on computed cost function. Fig 3 clearly depicts that the proposed protocol has longer stability period. This is expected, due to the appropriate selection of new forwarder in each round. Hence, each node consumes almost equal energy in each round and all the nodes die almost at the same time. In ATTEMPT, as temperature of forwarder nodes increases, nodes select alternate longer path which consumes more energy. Hence, these nodes die early. Our proposed protocol achieves $31 \%$ more stability period and $0.4 \%$ longer network lifetime.

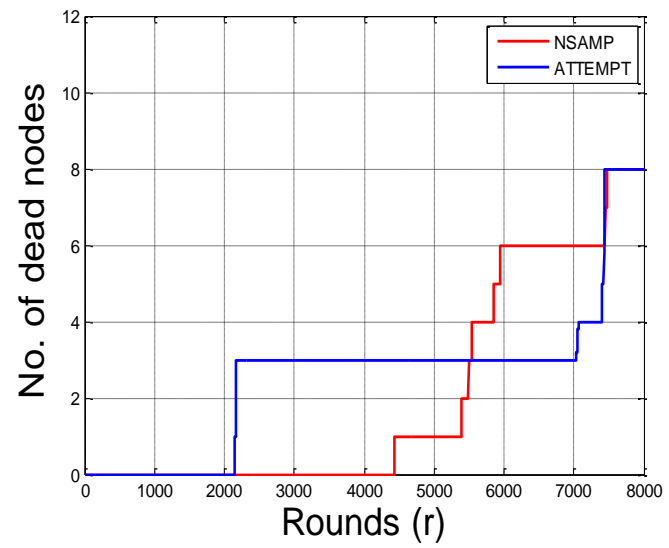

Fig. 3: Analysis of network lifetime

\section{B. Throughput}

Throughput is the successful packet received at the sink.As WBAN has critical and important data of patient, so itrequires a protocol which has minimum packet drop andmaximum successful data received at sink.NSAMP protocolachieves high throughput than ATTEMPT, as shown in fig6. Number of packets send to sink depends on the numberof alive nodes. More alive nodes send more packets to sinkwhich increases the throughput of network. The stabilityperiod of ATTEMPT is shorter than NSAMPprotocolwhich means number of packets sent to sink decreased.Hence, throughput of ATTEMPT decreased. On the hand,NSAMPprotocol achieves high throughput due to longerstability period.

\section{Residual energy}

The average energy of network consumed in each roundis presented in fig 5. The proposed model use multi hoptopology, in which each farthest node transmits its data tosink through a forwarder node. Forwarder node is electedusing aforementioned cost function. Selection of appropriateforwarder in each round contributes to save energy. To transfer packets to sink, our multi hop topology use different forwarder node in each round, this restricts over loading of particular node. 


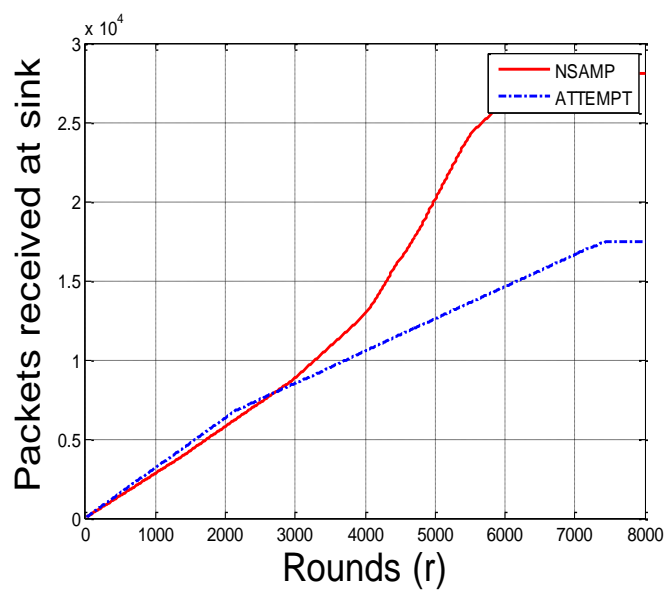

Fig. 4: Analysis of Throughput

Simulation results show that NSAMP protocol consumes minimum energy till $70 \%$ of simulation time. It means, in stability period, more nodes have enough energy and they transmit more data packet to sink. It also improves the throughput of the network. On the other hand, in ATTEMPT, some nodes exhaust early due to heavy traffic load.

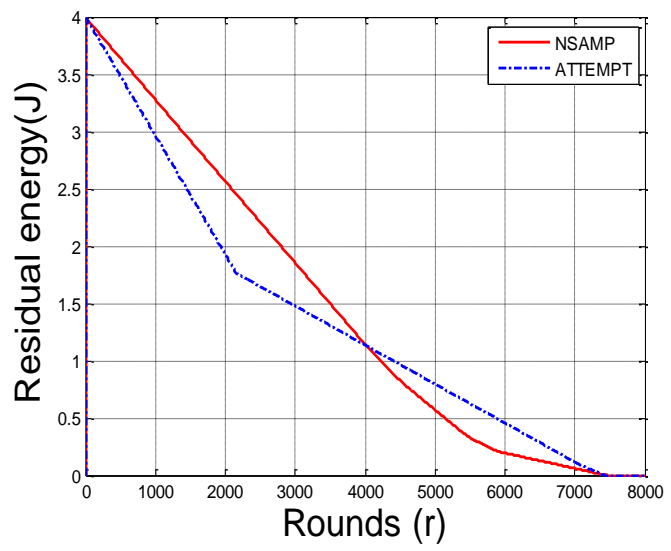

Fig. 5: Analysis of remaining energy

\section{Path Loss}

Fig 4 presents the path loss of different sensors. Path loss is a function of frequency and distance. Path loss shown in figure 6 is function of distance. It is calculated from its distance to sink with constant frequency 2.4GHz. We use path loss coefficient 3.38 and 4.1 for standard deviation

Proposed multi hop topology reduces the path loss as shown in figure 6. It is due to the fact that multi hop transmission reduces the distance, which leads to minimum path loss. Fig 6 represents the results of both topologies. Initially NSAMP protocol performs well. However, after 2000 rounds, path loss of ATTEMPT dramatically decreased because some nodes of ATTEMPT topology die. Minimum number of alive nodes has minimum cumulative path loss. As our proposed protocol has longer stability period and more alive nodes hasmore cumulative path loss. 


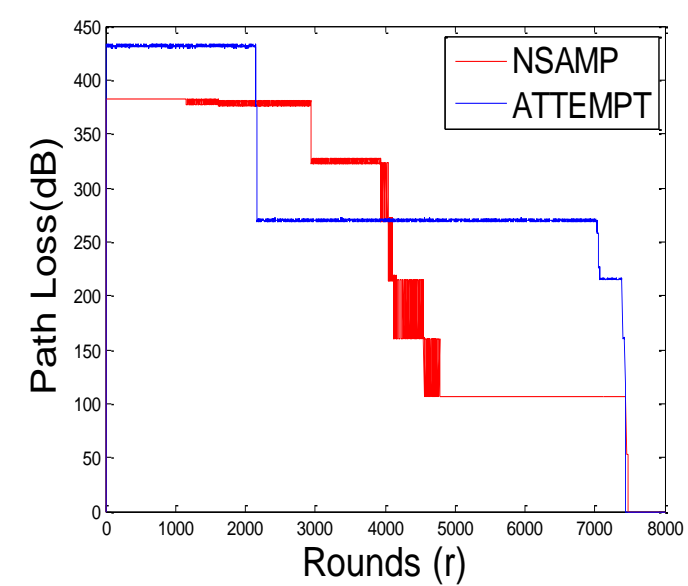

Fig. 6: network path loss

\section{E. Path loss model}

Path loss represents the signal attenuation and is measured in decibels (dB). Signal power is also degraded by Additive White Gaussian Noise (AWGN) [19]. Path loss is the difference between the transmitted power and received power whereas antenna gain may or may not be considered. Path loss occurs due to the increasing surface area of propagating wave front. Transmitting antenna radiates power outward and any object between transmitter and receiver causes destructionof radiated signal. In WBAN, different human postures, movement of body, hands and cloths, affects the transmitted signal. Path loss is related to the distance and frequency.

$$
\operatorname{PL}(f, d)=\operatorname{PL}(f) \times \operatorname{PL}(d)
$$

The relation of frequency with path loss is expressed as

$$
P L(f) \alpha f k
$$

Where $\mathrm{k}$ is frequency dependent factor and it is related to the geometry of the body. The relation of distance with path loss is given as

$$
\operatorname{PL}(\mathrm{f}, \mathrm{d})=\mathrm{PLo}+10 n \log 10 \mathrm{~d} / \mathrm{do}+\mathrm{X} \sigma
$$

Where PL is received power, $\mathrm{d}$ is the distance between transmitter and receiver, do is the reference distance, $\mathrm{n}$ is the path loss coefficient and its value depends on the propagation environment. In free space its value is 2, for WBAN, $\mathrm{n}$ varies from 3-4 for line of sight (LOS) communication and 5-7.4

fornon line of sight (NLOS) communication. $\mathrm{X}$ is Gaussian random variable and _ is standard deviation [21]. PLois received power at reference distance do and it is expressed as:

$$
\mathrm{PLo}=10 \log 10(4 \times \mathrm{d} \times \mathrm{f})^{2} / \mathrm{c}
$$

Where $\mathrm{f}$ is frequency, $\mathrm{c}$ speed of light and $\mathrm{d}$ is distance between transmitter and receiver. The value of reference distance do is $10 \mathrm{~cm}$. In reality it is difficult to predict strength of signal between transmitter and receiver boundary. To solvehis issue, we use a deviation variable $\mathrm{X} \sigma$.

\section{Conclusion}

In this paper, we propose a mechanism to route data in WBANs. The proposed schemes use a cost function to select appropriate route to sink. Cost function is calculated based on the residual energy of nodes and their distance from sink. Nodes with less value of cost function are elected as parentnode. Other nodes become the children of that parent node and forward their data to parent node. Two nodes for ECG and Glucose monitoring forward their data direct to sink asthey are placed near sink, also these two nodes can not beelected as parent node because both sensor node has criticaland important medical data. It is not required that these twonode deplete their energy in forwarding data of other nodes.Our simulation results shows that proposed routing schemeenhance the network stability time and packet delivered tosink. Path loss is also investigated in this protocol and in futurework, we will implement Expected Transmission Count (ETX)link metrics as demonstrated. 


\section{References}

[1] M. F. F. B. Ismail and L. W. Yie, “Acoustic monitoring system using wireless sensor networks,” Procedia Engineering, vol. 41, pp. 68-74, 2012.

[2] S.-D. Bao, Y.-T.Zhang, and L.-F. Shen, "Physiological signal based entity authentication for body area sensor networks and mobile healthcare systems," in Engineering in Medicine and Biology Society, 2005. IEEE-EMBS 2005.27th Annual International Conference of the, pp. 2455-2458, IEEE, 2005.

[3] R. Kaur, "Wireless body area network \& its application," 2011.

[4] L. Wang and H. Liang, "Research and improvement of the wireless sensor network routing algorithm gpsr," in Computing, Measurement, Control and Sensor Network (CMCSN), 2012 International Conference on, pp. 83-86, IEEE, 2012.

[5] "A survey on indoor localization techniques in wireless body area sensor networks,"

[6] S. Ullah, B. Shen, S. Riazul Islam, P. Khan, S. Saleem, and K. Sup Kwak, "A study of mac protocols for wbans,” Sensors, vol. 10, no. 1 , pp. $128-145,2009$.

[7] N. Timmons and W. Scanlon, "An adaptive energy efficient mac protocol for the medical body area network," in Wireless Communication, Vehicular Technology, Information Theory and Aerospace \&Electronic Systems Technology, 2009.Wireless VITAE 2009.1st International Conference on, pp. 587-593, IEEE, 2009.

[8] O. Omeni, A. Wong, A. J. Burdett, and C. Toumazou, "Energy efficient medium access protocol for wireless medical body area sensor networks," Biomedical Circuits and Systems, IEEE Transactions on, vol. 2, no. 4, pp. 251-259, 2008.

[9] S. J. Marinkovic, E. M. Popovici, C. Spagnol, S. Faul, and W. P. Marnane, "Energyefficient low duty cycle mac protocol for wireless body area networks," Information Technology in Biomedicine, IEEE Transactions on, vol. 13, no. 6, pp. 915-925,2009.

[10] G. Fang and E. Dutkiewicz, "Bodymac: Energy efficient tdma-based mac protocol for wireless body area networks," in Communications and Information Technology, 2009. ISCIT 2009.9th International Symposium on, pp. 1455-1459, IEEE, 2009.

[11] Guo, Cheng, R. Venkatesha Prasad, and Martin Jacobsson. "Packet forwarding with minimum energy consumption in body area sensor networks."Consumer Communications and Networking Conference (CCNC), 2010 7th IEEE.IEEE, 2010.

[12] Tsouri, Gill R., Adrian Sapio, and Jeff Wilczewski. "An investigation into relaying of creeping waves for reliable low-power body sensor networking." Biomedical Circuits and Systems, IEEE Transactions on 5.4 (2011): 307-319.

[13] Sapio, Adrian, and Gill R. Tsouri. "Low-power body sensor network for wireless ecg based on relaying of creeping waves at 2.4 ghz.” Body Sensor Networks (BSN), 2010 International Conference on. IEEE, 2010.

[14] N. Javaid, M. Yaqoob, M. Y. Khan, M. A. Khan, A. Javaid, Z. A.Khan, “Analyzing Delay in Wireless Multi-hop Heterogeneous Body Area Networks", Research Journal of Applied Sciences, Engineering and Technology, 2013.

[15] N. Javaid, I. Israr, M. A. Khan, A. Javaid, S. H. Bouk, Z. A. Khan, “Analyzing Medium Access Techniques in Wireless Body Area Networks", Research Journal of Applied Sciences, Engineering and Technology, 2013

[16] Quwaider, Muhannad, and SubirBiswas. "Probabilistic routing in onbody sensor networks with postural disconnections." Proceedings of the 7th ACM international symposium on Mobility management and wireless access.ACM, 2009.

[17] Maskooki, Arash, et al. "Opportunistic routing for body area network." Consumer Communications and Networking Conference (CCNC), 2011 IEEE.IEEE, 2011.

[18] Heinzelman, Wendi Rabiner, AnanthaChandrakasan, and HariBalakrishnan. "Energy-efficient communication protocol for wireless microsensor networks."System Sciences, 2000.Proceedings of the 33rd Annual Hawaii International Conference on.IEEE, 2000.

[19] Manzoor, B. et al. "Noise Filtering, Channel Modeling and Energy Utilization in Wireless Body Area Networks." High Performance Computing and Communication and 2012 IEEE 9th International Conference on Embedded Software and Systems (HPCC-ICESS), 2012 IEEE 14 ${ }^{\text {th }}$ International Conference on.IEEE, 2012. 